\title{
Associations of activity and sleep with quality of life: a Compositional Data \\ Analysis
}

Sanne Verhoog MSc, ${ }^{1,2}$ Kim V.E. Braun, RD, PhD, ${ }^{1}$ Arjola Bano, MD, PhD,,${ }^{1,2,3}$ Frank J.A. van Rooij, MSc, ${ }^{1}$ Oscar H. Franco, MD, PhD,,${ }^{1,2}$ Chantal M. Koolhaas, PhD, ${ }^{1}$ Trudy Voortman, $\mathrm{PhD}^{1}$

From the ${ }^{1}$ Department of Epidemiology, Erasmus University Medical Center, Rotterdam, The

Netherlands; ${ }^{2}$ Institute of Social and Preventive Medicine, University of Bern, Switzerland; and ${ }^{3}$ Department of Cardiology, Inselspital, Bern University Hospital, University of Bern, Switzerland

Address correspondence to: Trudy Voortman, PhD, Department of Epidemiology, Erasmus MC, Office Na-2716, PO Box 2040, 3000 CA Rotterdam, the Netherlands. E-mail: trudy.voortman@erasmusmc.nl. 
Introduction: Associations between time spent on physical activity, sedentary behavior, and sleep with quality of life are usually studied without considering that their combined time is fixed. This study investigates the reallocation of time spent on physical activity, sedentary behavior, and sleep during the 24-hour day and associations with quality of life.

Methods: Data from the 2011-2016 Rotterdam study were used to perform this cross-sectional analysis among 1,934 participants, aged 51-94 years. Time spent in activity levels (sedentary, light physical activity, moderate-to-vigorous physical activity, and sleep) were objectively measured with a wrist-worn accelerometer, combined with a sleep diary. Quality of life was measured using the EuroQoL 5D-3L questionnaire. The compositional isotemporal substitution method was used in 2018 to examine the association between the distribution of time spent in different activity behaviors and quality of life.

Results: Reallocation of 30 minutes from sedentary behavior, light physical activity, or sleep to moderate-to-vigorous physical activity was associated with higher quality of life, whereas reallocation from moderate-to-vigorous physical activity to sedentary behavior, light physical activity, or sleep was associated with lower quality of life. To illustrate, a reallocation of 30 minutes from sedentary behavior to moderate-to-vigorous physical activity was associated with a $3 \%(95 \% \mathrm{CI}=2,4)$ higher quality of life score. On the other hand, a reallocation of 30 minutes from moderate-to-vigorous physical activity to sedentary behavior was associated with a 4\% $(95 \% \mathrm{CI}=2,6)$ lower quality of life score.

Conclusions: Moderate-to-vigorous physical activity is important with regard to quality of life of middle-aged and elderly individuals. The benefits of preventing less time spent in moderateto-vigorous physical activity were greater than the benefits of more time spent in moderate-tovigorous physical activity. These results could shift the attention to interventions focused on 
preventing reductions in moderate-to-vigorous physical activity levels. Further longitudinal studies are needed to confirm these findings and explore causality. 


\section{INTRODUCTION}

The aging process is related to an increased risk of morbidity and disability, which could result in a lower quality of life (QoL). ${ }^{1}$ Recent studies have shown that higher levels of physical activity (PA) and lower levels of sedentary time are associated with better QoL. ${ }^{2,3}$ Additionally, both short and long sleep durations ( $\leq 6$ or $\geq 9$ hours) have been linked to poorer QoL. ${ }^{4,5}$ However, most previous studies on activity and QoL have important limitations.

First, the association of PA and sleep with QoL has been studied previously either in isolation or with only partial adjustment for time spent in other behaviors. ${ }^{6}$ Traditional regression and isotemporal substitution models use time-dependent activity data as absolute continuous values. However, daily time is constrained at 24 hours, and all activity combined contributes to a composite whole. Time spent in one level of activity necessarily replaces time spent in at least one other level, and little is known about the effect of replacing one level of activity with another in this finite space of time. Although some studies attempted to take this into account by expressing activity behaviors in proportions or percentages with respect to the given total, this still gives the problem of collinearity when assessing multiple behaviors. An alternative analytic approach is the compositional isotemporal substitution model, which addresses the codependency of activity behaviors to account for the time spent in each behavior by treating each behavior as a composite of a finite whole. ${ }^{7}$ Because the compositional analysis uses timedependent activity data as relative values, this also allows for asymmetrical results for the reallocation of behaviors. 
Second, measures of daytime activity and sleep in these studies have mostly been assessed with self-reported questionnaires. ${ }^{1,3-5}$ These subjective measurements are prone to reporting errors and recall bias, especially in older populations, in whom cognitive impairment is more likely. ${ }^{8}$ The Rotterdam Study is a large population-based study with objectively assessed measures of daytime activity and sleep in a middle-aged and elderly population, by using a wrist-worn triaxial accelerometer. ${ }^{9}$ Wrist-worn devices can be worn day and night, thereby allowing for collection of 24 hours of activity data. These 24-hour activity data and the compositional isotemporal substitution model can be used to assess the difference in QoL estimated for any reallocation between daily activity behaviors.

The aim of this study is to investigate the allocation of PA, sedentary behavior (SB) and sleep during the 24-hour day and its association with QoL in a middle-aged and elderly population of the Rotterdam Study.

\section{METHODS}

\section{Study Population}

A cross-sectional analysis was performed in 2018, embedded in the Rotterdam Study, an ongoing prospective population-based cohort study in the Netherlands. Detailed information on the Rotterdam Study can be found elsewhere. ${ }^{9}$

Between June 2011 and June 2014 (Wave 1) and between July 2014 and May 2016 (Wave 2), 3,507 participants were invited to wear an accelerometer for 7 days. Of this total, 2,102 participants had complete information on QoL and data on activity for $\geq 4$ days with $>1,200$ 
minutes/day (Figure 1). From this group, an additional 79 participants without sleep diary data were excluded and 89 observations were excluded for participants who participated in both waves. The remaining 1,934 participants were considered eligible for analysis. There were no major differences between the included and excluded participants (Appendix Table 1).

\section{Measures}

To measure activity, all participants were asked to wear a triaxial accelerometer (GeneActiv) on the non-dominant wrist for 7 consecutive days and nights, and to additionally complete a 7-day sleep diary in which overnight sleep periods were reported. As the GeneActiv device is waterproof, it can also be worn while bathing and swimming. As in previous studies, the accelerometer was sampled at $50 \mathrm{~Hz}$ and acceleration was expressed in milligal (mg) relative to gravity $\left(1 \mathrm{~g}=9.81 \mathrm{~m} / \mathrm{s}^{2}\right) .{ }^{10-13}$ Non-wear time was estimated as time periods where the SD of acceleration in all three axes fell below $13 \mathrm{mg}$ for $>1$ hour, and excluded from analyses. Accelerometer data were processed in Python version 2.6.6 using the open-access PAMPRO software, version 0.3 (T. White (2016): https://zenodo.org/badge/latestdoi/18706328).

Activity was categorized into SB ( $<48 \mathrm{mg}$ ), light-intensity PA (LIPA; 48-154 mg), and moderate-to-vigorous PA (MVPA; $>154 \mathrm{mg}) .{ }^{14}$ Sleep duration was quantified with a validated algorithm, ${ }^{15}$ using information on overnight sleep periods from the sleep diary, and subtracted from total SB. All four activity levels were expressed in minutes/day and add up to 24 hours when combined.

Health-related QoL (hereafter referred to as QoL) was measured using the Dutch version of the EuroQoL 5D-3L (EQ-5D-3L). The EQ-5D-3L consists of five dimensions (5D; i.e., mobility, 
self-care, daily activities, pain/discomfort, and mood) assessed with a single question. ${ }^{16}$ These five questions can be scored according to three response levels (3L; i.e., no problems, some or moderate problems, and extreme problems) coded from 1 to 3 . Placing the answers of all five questions in a series results in a five-digit QoL profile (e.g., 12323), whereby 11111 represents the best possible QoL and 33333 the worst. This profile was converted with weighted utility scores to an overall index score, ranging from 0 (poor QoL) to 1 (perfect QoL), as described in more detail elsewhere. ${ }^{17}$ As an additional indicator of QoL, the EQ-5D-3L includes a standard vertical $20 \mathrm{~cm}$ visual analogue scale (VAS), where individuals can indicate their current health on a scale from 0 to 100 .

Information on covariates was collected through home interviews, questionnaires, or at the study center. Height and weight were measured and BMI was calculated $\left(\mathrm{kg} / \mathrm{m}^{2}\right)$. A food frequency questionnaire was used to compute a diet quality score ${ }^{18}$ and alcohol consumption (g/day) was obtained. In a home interview, the authors obtained information on smoking (former/current/never), education (four categories ${ }^{19}$ ), marital status (partner yes/no), job status (employed/unemployed), and living situation (completely independent/partially independent). Information on chronic diseases was obtained from the interview, measurements in the center, and medical records. A comorbidity score was created as the number of current chronic conditions (i.e., presence of cancer, diabetes, or cardiovascular disease). Information on the use of sleep medication (yes/no) in the past 7 days was obtained from the sleep diary.

\section{Statistical Analysis}

Descriptive statistics were used to characterize the sample. For the compositional analysis, activity compositions were created by expressing time spent in each activity (i.e., LIPA, MVPA, 
$\mathrm{SB}$, and sleep) as a proportion of the 24-hour day. The activity compositions were then expressed as isometric log ratio (ilr) coordinates to account for interdependency of the activity domains. ${ }^{7}$ These coordinates were used to express the activity composition in linear regression models, with the QoL score as the dependent variable and the activity composition as the independent variable. The linear models were then used in a prediction function in $\mathrm{R}$, to estimate the $\mathrm{QoL}$ score for a specific activity composition. In this scenario, first the QoL score for the mean activity composition of the study population was obtained and then for a composition in which time spent in one activity domain was substituted for time spent in another (e.g., 30 minutes of sleep reallocating 30 minutes of SB) relative to the mean activity composition. Then the difference in QoL scores along with the 95\% CI was computed.

Two models were created. The first model was adjusted for sex and age. The second model was additionally adjusted for education, marital status, living situation, job status, smoking, alcohol, diet score, BMI, comorbidity score, and use of sleep medication. ${ }^{20,21}$ Within each model, two different analyses were performed. In the first analysis, the differences in the EQ-5D-3L index score were estimated. The second analysis estimated the differences in the VAS score.

The data contained $43.7 \%$ missing data for dietary variables and $6.6 \%$ for job status. All other covariates had $<2 \%$ missing data. Multiple imputation ( $n=10$ imputations) was used by the expectation maximization method. Analyses were performed on all imputed data sets separately and the results were pooled. Stratified analyses by sex, age, and MVPA level were conducted, whereby age and MVPA were stratified at the median level (i.e., 72 years and 79 minutes, respectively). ${ }^{20,21}$ All analyses were conducted using SPSS, version 24 and R, version 3.1.3. 


\section{RESULTS}

Characteristics of the study population are presented in Table 1 . The mean age of the participants was $70.9(\mathrm{SD}=9.27)$ years and $51.5 \%$ were women. Mean hours of sleep and SB per day were $6.43(\mathrm{SD}=1.04)$ hours and $13.54(\mathrm{SD}=1.31)$ hours, respectively. Mean minutes of LIPA and MVPA per day were $148.11(\mathrm{SD}=31.54)$ minutes and $82.72(\mathrm{SD}=28.74)$ minutes, respectively. The mean QoL EQ-5D-3L index score was $0.87(\mathrm{SD}=0.16)$.

In the multivariable-adjusted models, a reallocation of 30 minutes from MVPA to SB, LIPA, or sleep was associated with a lower EQ-5D-3L index score (Table 2). Similarly, reallocation of 30 minutes to MPVA from SB, LIPA, or sleep was associated with a higher EQ-5D-3L index score. The largest estimated difference in EQ-5D-3L index score was $-0.05(95 \% \mathrm{CI}=-0.08,-0.02)$ on a score ranging from 0 to 1 , when reallocating 30 minutes of MVPA to LIPA, meaning that this reallocation was associated with a 5\% decrease in EQ-5D-3L index score. The estimated differences in EQ-5D-3L index score were not exactly symmetrical. For example, a reallocation of 30 minutes from MPVA to SB was associated with a slightly larger difference in EQ-5D-3L index score $(-0.04,95 \% \mathrm{CI}=-0.06,-0.02)$ than when 30 minutes were reallocated from SB to $\operatorname{MVPA}(0.03,95 \% \mathrm{CI}=0.02,0.04)$.

For the VAS score, the results were similar to those for the EQ-5D-3L index score (Table 2). Reallocating time from MVPA was associated with a lower VAS score, whereas reallocating time to MVPA was associated with a higher VAS score. For the VAS score, the largest estimated 
difference was $-3.84(95 \% \mathrm{CI}=-6.35,-1.32)$ on a score ranging from 0 to 100 , for reallocation of 30 minutes from MVPA to LIPA.

When 30 minutes of SB or sleep were replaced with 30 minutes of LIPA, or vice versa, the association was not significant with both indicators of QoL (Table 2). Results of the sex-age adjusted model were similar for the EQ-5D-3L index score and VAS score (Appendix Table 2).

In the stratified analyses, associations for MVPA were slightly stronger for men, among participants aged $\geq 72$ years, and among those with lower MVPA levels, but remained statistically significant in all subgroups for the EQ-5DL-3L index (Appendix Tables 3-5). Among men, younger participants, and those with high MVPA levels, a higher LIPA tended to be associated with a lower QoL, but only for the EQ-5D-3L index score and estimates were small. For QoL measured with the VAS score, results for MVPA were in similar direction, but no longer statistically significant for women, participants aged $<72$ years, and those with higher MVPA levels (Appendix Tables 6-8).

\section{DISCUSSION}

In this middle-aged and elderly population, reallocating 30 minutes to MVPA from any other activity behavior is associated with a higher QoL score, whereas reallocating 30 minutes from MVPA to any other activity behavior is associated with a lower QoL score. Additionally, the association between MVPA and QoL is not exactly symmetrical; a decrease in MVPA has a slightly larger association than an increase in MVPA. 
To the authors' knowledge, this is the first study using a compositional data analysis to investigate the association between the distribution of time spent in four different activity behaviors and QoL in middle-aged and elderly adults. Recently, a similar study using compositional data analysis of activities in relation to QoL was conducted among children. ${ }^{22}$ This study also found that increasing time spent MVPA was associated with a higher QoL and decreasing time spent in MVPA with a lower QoL. Another study examined the association of SB and PA with self-rated health, and found that substituting sedentary time with an equal amount of LIPA or MVPA was associated with better physical health. ${ }^{23}$

A systematic review reported a consistently positive association between PA and QoL. ${ }^{24}$ Individuals who spend more time in MVPA may report a better QoL because of the effects on physical health (e.g., reduced disease risk and improved fitness) and psychological benefits of physical activity (e.g., improved self-esteem and socialization). ${ }^{25,26}$ Although no conclusions on the temporal direction or causality of the association can be drawn from this study, a recent meta-analysis of exercise interventions on QoL showed that over a period of 3-6 months, a small but meaningful improvement in QoL can be achieved by exercise interventions in a healthy population, which suggest a causal effect. ${ }^{27}$ Still, the reverse might also be true, as a better QoL may be expected to affect activity levels. A study investigating the factors associated with physical activity in older adults reported QoL as one of the most important factors associated with engagement in $\mathrm{PA}^{28}$

Findings from this study suggest that the association of replacing MVPA with another behavior and vice versa with QoL is not exactly symmetrical. For example, 30 minutes less MVPA and 
more SB were associated with a 4\% decrease in EQ-5D-3L index score, whereas replacing 30 minutes more MVPA and less SB were associated with a 3\% increase EQ-5D-3L index score. This asymmetry was also found in other studies using the compositional data analysis method. ${ }^{22,29}$ This asymmetry seems plausible as removing 30 minutes from MVPA is a large amount of daily time spent in MVPA, whereas decreasing SB by 30 minutes only accounts for $2 \%-5 \%$ change in total SB time.

These findings did not show a significant association when SB or sleep was replaced with LIPA and vice versa. A similar study examined the effects of replacing SB with PA on health-related QoL and found that LIPA was unrelated to QoL. ${ }^{30}$ However, a recent systematic review reported that LIPA is beneficially associated with several health outcomes, including well-being, after adjustment for MVPA. ${ }^{31}$ An explanation for this contradicting finding could be that LIPA includes more household chores (e.g., cooking, cleaning, ironing), as compared with MVPA, which includes activities that typically take place in leisure settings. ${ }^{32}$ Further research exploring whether and to what extent LIPA contributes to QoL may provide insights to this issue.

In this study, associations were somewhat stronger among men, those at a higher age, and those with lower overall MVPA levels, but directions were the same in all subgroups. Other crosssectional studies also found that higher levels of PA were positively associated with QoL for both men and women. ${ }^{21,33}$ A literature review on PA and QoL reported that there was no evidence across studies that age was a moderator of QoL, which is in line with the present findings. ${ }^{34}$ These findings support that increasing MVPA may have a slightly greater benefit among those who are less physically active, although the difference with those who were more 
physically active in the study population was small. A difference in associations of MVPA and QoL measured with the EQ-5D-3L index score or the VAS score was not found. This suggests that the combined index score of QoL based on five different domains reflects an individual's current health at the moment of filling in the questionnaire indicated on a visual analogue scale, which has been reported in previous literature. ${ }^{35}$

This study may have implications for public health, suggesting that the associated harm of less MVPA is greater than the benefit of more MVPA, relative to the mean activity composition of this study population. The mean time spent in MVPA in this study was 82.7 minutes per day, which far exceeds the 150 minutes per week recommendation from the WHO. ${ }^{36}$ For individuals who already have high levels of PA, interventions focused on preventing a decline rather than increasing PA could therefore already be effective. This could highlight the need for interventions focused around events or occasions when time spent in MVPA typically tends to decline (e.g., during the colder months of the year, in certain life stages, or after specific life events). ${ }^{37}$ Most current interventions or public health campaigns are aimed at increasing PA levels, but very few specifically aim to support individuals to remain active through the life course.

One of the major strengths of this study is the use of the compositional data analysis methods, which accounts for the constraint nature of time. Another strength is the large sample size of 1,934 participants, from a well-characterized population-based cohort. Furthermore, data on activities were measured objectively with waterproof accelerometers worn over the 24 hours of a day, which ensured high compliance. Participants were requested to wear the accelerometer for 7 
consecutive days, which exceeds the $3-5$ days required to assess a daily estimate of the individual's habitual activity. ${ }^{38}$ Moreover, it has been reported that activity at middle and elderly age tends to be relatively constant with time. ${ }^{39}$

\section{Limitations}

The findings of this study must be considered in the context of some limitations. First, the crosssectional analyses preclude any assessment of directionality of the association. Second, this study used the $3 \mathrm{~L}$ version of the EQ-5D questionnaire. A 5L version is also available comprising five levels of answering options, which could therefore be a more comprehensive measurement of QoL with a smaller ceiling effect. ${ }^{40}$ Furthermore, the EQ-5D-3L instrument only assesses healthrelated QoL. However, the findings from the index score and VAS score were similar, suggesting that the measurement of QoL was robust. Accelerometers do not allow differentiation between the postural allocations of sitting, standing and lying down, and can therefore not distinguish different types of activity. Furthermore, only overnight sleep periods were included from the sleep diary. Therefore, naps during the day are not taken into account and included as SB. Finally, the categorization of activity levels with accelerometry is dependent on the algorithm and cut points used, and should therefore be interpreted with caution.

\section{CONCLUSIONS}

In summary, MVPA is an important activity in regard to the QoL of middle-aged and elderly individuals. The associated benefits of preventing 30 minutes less MVPA are greater than the benefits of 30 minutes more MVPA. These results could shift the attention of interventions to not only focusing on increasing MVPA but also preventing a decline in a middle-aged and elderly 
population. However, further longitudinal studies are needed to confirm these findings and the availability of repeated measurements would contribute to elucidate the directionality of the observed associations. 


\section{ACKNOWLEDGMENTS}

The authors are grateful to the study participants, the staff from the Rotterdam Study, and the participating general practitioners and pharmacists. We thank Amy Hofman for her help with additional data analysis.

The Rotterdam Study is funded by Erasmus MC and Erasmus University, Rotterdam, the Netherlands; the Netherlands Organisation for Scientific Research; the Netherlands Organisation for the Health Research and Development; the Research Institute for Diseases in the Elderly; the Ministry of Education, Culture and Science; the Ministry for Health, Welfare and Sports; the European Commission, and the Municipality of Rotterdam.

All procedures were performed in accordance with the 1964 Helsinki declaration and its later amendments and the Rotterdam Study was approved by the IRB (Medical Ethics Committee) of Erasmus Medical Center (registration number MEC 02.1015) and by the Dutch Ministry of Health, Welfare and Sport (Population Screening Act WBO, license number 1071272-159521PG). All participants provided written informed consent to participate in the study and to have their information obtained from treating physicians. The Rotterdam Study is registered in the Netherlands National Trial Register (www.trialregister.nl) and into the WHO International Clinical Trials Registry Platform (www.who.int/ictrp/network/primary/en/) under shared catalogue number NTR6831). 
Data can be obtained on request. Requests should be directed toward the management team of the Rotterdam Study (secretariat.epi@erasmusmc.nl), which has a protocol for approving data requests. Because of restrictions based on privacy regulations and informed consent of the participants, data cannot be made freely available in a public repository.

OHF and CMK had the original idea for the study. SV and CMK performed the statistical analyses and interpreted the data. SV drafted the article. All authors revised the manuscript critically for important intellectual content and approved the final article.

No financial disclosures were reported by the authors of this paper. 


\section{REFERENCES}

1. Brown DS, Thompson WW, Zack MM, Arnold SE, Barile JP. Associations between health-related quality of life and mortality in older adults. Prev Sci. 2015;16:21-30. https://doi.org/10.1007/s11121-013-0437-z.

2. Anokye NK, Trueman P, Green C, Pavey TG, Taylor RS. Physical activity and health related quality of life. BMC Public Health. 2012;12:624. https://doi.org/10.1186/14712458-12-624.

3. Balboa-Castillo T, Leon-Munoz LM, Graciani A, Rodriguez-Artalejo F, GuallarCastillon P. Longitudinal association of physical activity and sedentary behavior during leisure time with health-related quality of life in community-dwelling older adults. Health Qual Life Outcomes. 2011;9:47. https://doi.org/10.1186/1477-7525-9-47.

4. Faubel R, Lopez-Garcia E, Guallar-Castillón P, et al. Sleep duration and health-related quality of life among older adults: a population-based cohort in Spain. Sleep. 2009;32(8):1059-1068. https://doi.org/10.1093/sleep/32.8.1059.

5. Magee CA, Caputi P, Iverson DC. Relationships between self-rated health, quality of life and sleep duration in middle aged and elderly Australians. Sleep Med. 2011;12(4):346350. https://doi.org/10.1016/i.sleep.2010.09.013.

6. Pedišić Ž. Measurement issues and poor adjustments for physical activity and sleep undermine sedentary behaviour research - the focus should shift to the balance between sleep, sedentary behaviour, standing and activity. Kinesiology (Zagreb). 2014;46(1):135146. 
7. Dumuid D, Pedišić Ž, Stanford TE, et al. The compositional isotemporal substitution model: a method for estimating changes in a health outcome for reallocation of time between sleep, physical activity and sedentary behaviour. Stat Methods Med Res. 2019;28(3):846-857. https://doi.org/10.1177/0962280217737805.

8. Rait G, Fletcher A, Smeeth L, et al. Prevalence of cognitive impairment: results from the MRC trial of assessment and management of older people in the community. Age Ageing. 2005;34(3):242-248. https://doi.org/10.1093/ageing/afi039.

9. Ikram MA, Brusselle GGO, Murad SD, et al. The Rotterdam Study: 2018 update on objectives, design and main results. Eur J Epidemiol. 2017;32(9):807-850. https://doi.org/10.1007/s10654-017-0321-4.

10. da Silva ICM, van Hees VT, Ramires VV, et al. Physical activity levels in three Brazilian birth cohorts as assessed with raw triaxial wrist accelerometry. Int J Epidemiol. 2014;43(6):1959-1968. https://doi.org/10.1093/ije/dyu203.

11. Hildebrand M, Van Hees VT, Hansen BH, Ekelund U. Age group comparability of raw accelerometer output from wrist- and hip-worn monitors. Med Sci Sports Exerc. 2014;46(9):1816-1824. https://doi.org/10.1249/mss.0000000000000289.

12. Koolhaas CM, van Rooij FJA, Schoufour JD, et al. Objective measures of activity in the elderly: distribution and associations with demographic and health factors. J Am Med Dir Assoc. 2017;18(10):838-847. https://doi.org/10.1016/j.jamda.2017.04.017.

13. Sabia S, van Hees VT, Shipley MJ, et al. Association between questionnaire- and accelerometer-assessed physical activity: the role of sociodemographic factors. Am J Epidemiol. 2014;179(6):781-790. https://doi.org/10.1093/aje/kwt330. 
14. White T, Westgate K, Wareham NJ, Brage S. Estimation of physical activity energy expenditure during free-living from wrist accelerometry in UK adults. PLoS One. 2016;11(12):e0167472. https://doi.org/10.1371/journal.pone.0167472.

15. van Hees VT, Sabia S, Anderson KN, et al. A novel, open access method to assess sleep duration using a wrist-worn accelerometer. PLoS One. 2015;10(11):e0142533. https://doi.org/10.1371/journal.pone.0142533.

16. The EuroQol Group. EuroQol--a new facility for the measurement of health-related quality of life. Health Policy. 1990;16(3):199-208. https://doi.org/10.1016/0168$\underline{8510(90) 90421-9 .}$.

17. Lamers LM, McDonnell J, Stalmeier PF, Krabbe PF, Busschbach JJ. The Dutch tariff: results and arguments for an effective design for national EQ-5D valuation studies. Health Econ. 2006;15(10):1121-1132. https://doi.org/10.1002/hec.1124.

18. Voortman T, Kiefte-de Jong JC, Ikram MA, et al. Adherence to the 2015 Dutch dietary guidelines and risk of non-communicable diseases and mortality in the Rotterdam Study. Eur J Epidemiol. 2017;32(11):993-1005. https://doi.org/10.1007/s10654-017-0295-2.

19. UNESCO Institute for Statistics. International standard classification of education: ISCED 2011. UNESCO Institute for Statistics Montreal; 2012. https://doi.org/10.15220/978-92-9189-123-8-en.

20. Koolhaas CM, Dhana K, van Rooij FJA, Schoufour JD, Hofman A, Franco OH. Physical activity types and health-related quality of life among middle-aged and elderly adults: the Rotterdam Study. J Nutr Health Aging. 2018;22(2):246-253. https://doi.org/10.1007/s12603-017-0902-7. 
21. Vuillemin A, Boini S, Bertrais S, et al. Leisure time physical activity and health-related quality of life. Prev Med. 2005;41(2):562-569. https://doi.org/10.1016/j.ypmed.2005.01.006.

22. Dumuid D, Maher C, Lewis LK, et al. Human development index, children's healthrelated quality of life and movement behaviors: a compositional data analysis. Qual Life Res. 2018;27(6):1473-1482. https://doi.org/10.1007/s11136-018-1791-x.

23. Buman MP, Hekler EB, Haskell WL, et al. Objective light-intensity physical activity associations with rated health in older adults. Am J Epidemiol. 2010;172(10):1155-1165. https://doi.org/10.1093/aje/kwq249.

24. Bize R, Johnson JA, Plotnikoff RC. Physical activity level and health-related quality of life in the general adult population: a systematic review. Prev Med. 2007;45(6):401-415. https://doi.org/10.1016/j.ypmed.2007.07.017.

25. Elavsky S, McAuley E, Motl RW, et al. Physical activity enhances long-term quality of life in older adults: efficacy, esteem, and affective influences. Ann Behav Med. 2005;30(2):138-145. https://doi.org/10.1207/s15324796abm3002_6.

26. Gill DL, Hammond CC, Reifsteck EJ, et al. Physical activity and quality of life. J Prev Med Public Health. 2013;46(suppl 1):S28-S34. https://doi.org/10.3961/jpmph.2013.46.s.s28.

27. Gillison FB, Skevington SM, Sato A, Standage M, Evangelidou S. The effects of exercise interventions on quality of life in clinical and healthy populations; a meta-analysis. Soc Sci Med. 2009;68(9):1700-1710. https://doi.org/10.1016/j.socscimed.2009.02.028. 
28. McKee G, Kearney PM, Kenny RA. The factors associated with self-reported physical activity in older adults living in the community. Age Ageing. 2015;44(4):586-592. https://doi.org/10.1093/ageing/afv042.

29. Chastin SFM, Palarea-Albaladejo J, Dontje ML, Skelton DA. Combined effects of time spent in physical activity, sedentary behaviors and sleep on obesity and cardio-metabolic health markers: a novel compositional data analysis approach. PLoS One. 2015;10(10):e0139984. https://doi.org/10.1371/journal.pone.0139984.

30. Yasunaga A, Shibata A, Ishii K, et al. Replacing sedentary time with physical activity: effects on health-related quality of life in older Japanese adults. Health Qual Life Outcomes. 2018;16:240. https://doi.org/10.1186/s12955-018-1067-8.

31. Amagasa S, Machida M, Fukushima N, et al. Is objectively measured light-intensity physical activity associated with health outcomes after adjustment for moderate-tovigorous physical activity in adults? A systematic review. Int J Behav Nutr Phys Act. 2018;15:65. https://doi.org/10.1186/s12966-018-0695-z.

32. Ainsworth BE, Haskell WL, Herrmann SD, et al. 2011 Compendium of Physical Activities: a second update of codes and MET values. Med Sci Sports Exerc. 2011;43(8):1575-1581. https://doi.org/10.1249/mss.0b013e31821ece12.

33. Wendel-Vos GC, Schuit AJ, Tijhuis MA, Kromhout D. Leisure time physical activity and health-related quality of life: cross-sectional and longitudinal associations. Qual Life Res. 2004;13(3):667-677. https://doi.org/10.1023/b:qure.0000021313.51397.33.

34. Rejeski WJ, Mihalko SL. Physical activity and quality of life in older adults. $J$ Gerontol A Biol Sci Med Sci. 2001;56(suppl 2):23-35. https://doi.org/10.1093/gerona/56.suppl_2.23. 
35. Whynes DK. Correspondence between EQ-5D health state classifications and EQ VAS scores. Health Qual Life Outcomes. 2008;6:94. https://doi.org/10.1186/1477-7525-6-94.

36. WHO. Global recommendations on physical activity for health. Geneva: WHO; 2010.

37. Aspvik NP, Viken H, Ingebrigtsen JE, et al. Do weather changes influence physical activity level among older adults? - The Generation 100 Study. PloS One. 2018;13(7):e0199463. https://doi.org/10.1371/journal.pone.0199463.

38. Trost SG, McIver KL, Pate RR. Conducting accelerometer-based activity assessments in field-based research. Med Sci Sports Exerc. 2005;37(11 suppl):S531-S543. https://doi.org/10.1249/01.mss.0000185657.86065.98.

39. Caspersen CJ, Pereira MA, Curran KM. Changes in physical activity patterns in the United States, by sex and cross-sectional age. Med Sci Sports Exerc. 2000;32(9):16011609. https://doi.org/10.1097/00005768-200009000-00013.

40. Kim SH, Kim HJ, Lee SI, Jo MW. Comparing the psychometric properties of the EQ-5D3L and EQ-5D-5L in cancer patients in Korea. Qual Life Res. 2012;21(6):1065-1073. https://doi.org/10.1007/s11136-011-0018-1. 


\section{LIST OF FIGURES}

Figure 1. Flow chart of participant inclusion. 
Table 1. Characteristics of the Study Population $(n=1,934)$

\begin{tabular}{|c|c|}
\hline Characteristics & Mean (SD) \\
\hline Age, years & $70.9(9.27)$ \\
\hline Women, n (\%) & $996(51.5)$ \\
\hline \multicolumn{2}{|l|}{ Educational level, n (\%) } \\
\hline Primary education & $118(6.1)$ \\
\hline Lower education & $719(37.2)$ \\
\hline Intermediate education & $597(30.9)$ \\
\hline Higher education & $500(25.9)$ \\
\hline Living independent, $\mathrm{n}(\%)$ & $1,842(95.2)$ \\
\hline Employed, n (\%) & $426(22.0)$ \\
\hline Living with someone, $\mathrm{n}(\%)$ & $1,397(72.2)$ \\
\hline \multicolumn{2}{|l|}{ Smoking, n (\%) } \\
\hline Never & $627(32.4)$ \\
\hline Former & $1,137(58.8)$ \\
\hline Current & $170(8.8)$ \\
\hline Alcohol, grams/day & $12.9(15.7)$ \\
\hline BMI & $25.1(4.6)$ \\
\hline Diet quality score $(0-14)$ & $6.8(1.9)$ \\
\hline \multicolumn{2}{|l|}{ Comorbidities, n (\%) } \\
\hline 0 & $1,138(58.8)$ \\
\hline 1 & $623(32.2)$ \\
\hline 2 & $153(7.9)$ \\
\hline 3 & $20(1.0)$ \\
\hline Use of sleep medication, $\mathrm{n}(\%)$ & $283(14.6)$ \\
\hline Sleep (hours/day) & $6.4(1.0)$ \\
\hline Sedentary behavior (hours/day) & $13.5(1.3)$ \\
\hline LIPA (minutes/day) & $148.1(31.5)$ \\
\hline MVPA (minutes/day) & $82.7(28.7)$ \\
\hline EQ-5D-3L index score & $0.87(0.16)$ \\
\hline VAS score & $78.9(14.1)$ \\
\hline
\end{tabular}

Notes: Numbers are mean (SD), unless otherwise stated.

LIPA, light intensity physical activity; MVPA, moderate-to-vigorous physical activity; VAS, visual analogue scale. 
Table 2. Associations of 30 Minutes Reallocations Between Activity Behaviors With the EQ-5D-3L Index Score and VAS Score $(\mathrm{n}=1,934)$

\begin{tabular}{|c|c|c|c|c|}
\hline Variable & LIPA & MVPA & SB & Sleep \\
\hline & Delta (95\% CI) & Delta (95\% CI) & Delta (95\% CI) & Delta (95\% CI) \\
\hline \multicolumn{5}{|c|}{ EQ-5D-3L index score } \\
\hline LIPA & & $-0.05(-0.08,-0.02)$ & $-0.01(-0.02,0.001)$ & $-0.01(-0.02,0.002)$ \\
\hline MVPA & $0.04(0.02,0.06)$ & & $0.03(0.02,0.04)$ & $0.03(0.02,0.04)$ \\
\hline SB & $0.01(-0.001,0.03)$ & $-0.04(-0.06,-0.02)$ & & $0.002(-0.002,0.005)$ \\
\hline Sleep & $0.01(-0.002,0.02)$ & $-0.04(-0.06,-0.02)$ & $-0.001(-0.005,0.002)$ & \\
\hline \multicolumn{5}{|l|}{ VAS score } \\
\hline LIPA & & $-3.84(-6.35,-1.32)$ & $-0.86(-1.84,0.12)$ & $-0.66(-1.63,0.31)$ \\
\hline MVPA & $3.05(0.87,5.24)$ & & $2.00(0.89,3.11)$ & $2.20(1.05,3.36)$ \\
\hline SB & $1.05(-0.13,2.24)$ & $-2.97(-4.63,-1.32)$ & & $0.20(-0.09,0.50)$ \\
\hline Sleep & $0.86(-0.31,2.04)$ & $-3.16(-4.86,-1.47)$ & $-0.19(-0.47,0.09)$ & \\
\hline
\end{tabular}

Note: Boldface indicates statistical significance $(p<0.05)$. Estimates are obtained from compositional data analysis and reflect difference in EQ-5D-3L index score and VAS score for the reallocation of time from the behavior in the column to the behavior in the row, while keeping the time spent in other activities constant (i.e., the first value of -0.05 in row 1 is the estimated difference in EQ5D-3L index score for the reallocation of 30 minutes from MVPA to LIPA). Reallocations of behaviors are relative to the mean activity composition of the study population. Higher EQ-5D-3L index score and VAS score indicate higher quality of life. The effect is computed for time reallocation around the average composition. Models are adjusted for age, sex, educational status, marital status, living situation, job status, smoking, alcohol intake, dietary guidelines score, BMI, comorbidity score and the use of sleep medication.

LIPA, light intensity physical activity; MVPA, moderate-to-vigorous physical activity; SB, sedentary behavior; VAS, visual analogue scale. 
3,507 Participants invited to wear an accelerometer between June 2011 and June 2014 (wave 1) and between July 2014 and May 2016 (wave 2), of which 482 participants were invited twice

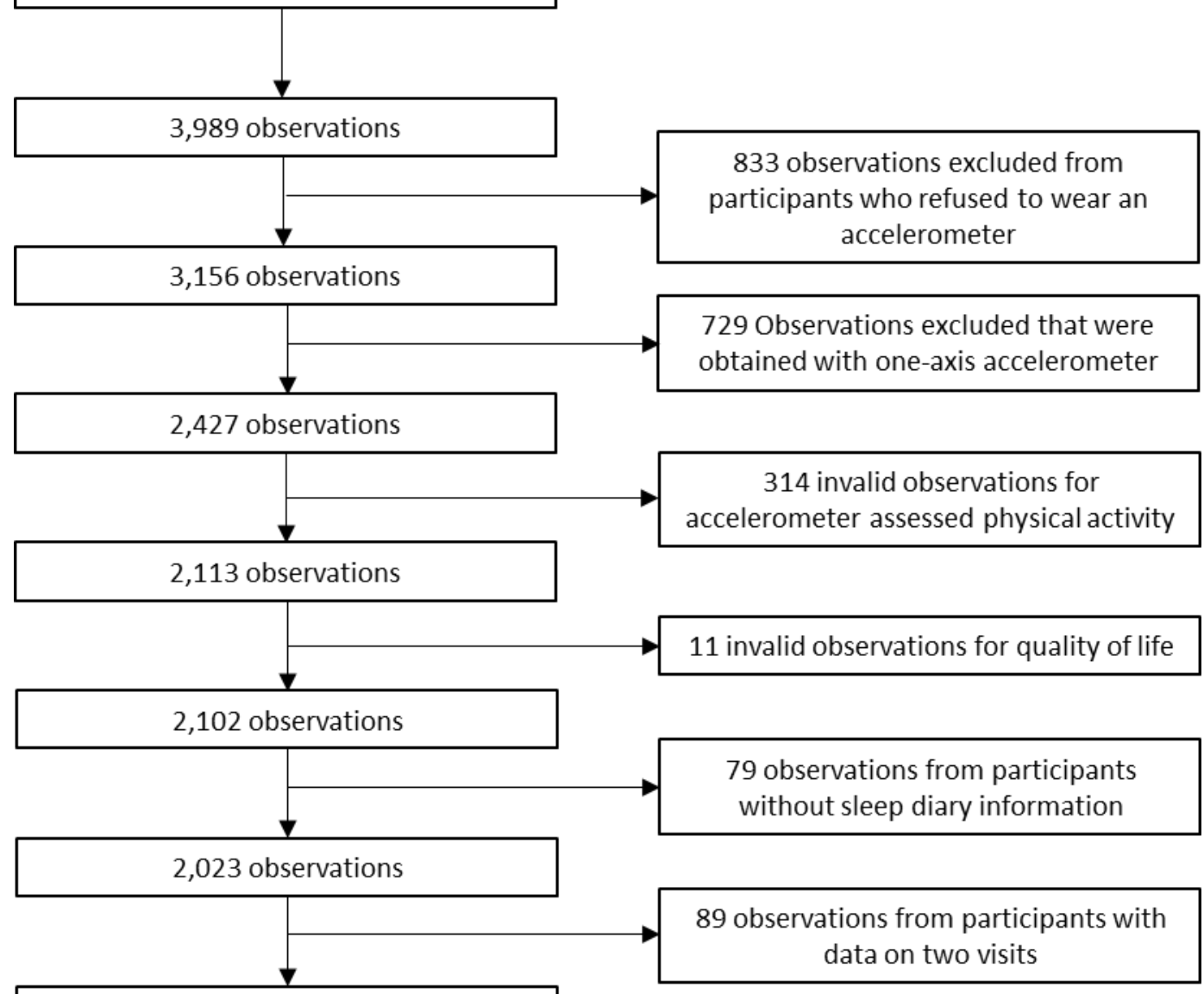

1,934 observations included in the analyses 
Appendix Table 1. Characteristics of the Included and Excluded Participants

\begin{tabular}{lcc}
\hline Characteristics & Included (N=1,934) & Excluded (N=1,573) \\
\hline Age, years & $70.9(9.27)$ & $71.2(10.1)$ \\
Women, n (\%) & $996(51.5)$ & $961(61.1)$ \\
Educational level, n (\%) & & \\
$\quad$ Primary education & $116(6.0)$ & $128(8.1)$ \\
$\quad$ Lower education & $712(36.8)$ & $670(42.6)$ \\
$\quad$ Intermediate education & $589(30.5)$ & $462(29.4)$ \\
$\quad$ Higher education & $493(25.5)$ & $292(18.6)$ \\
Living independent, n (\%) & $1,841(95.2)$ & $1,460(92.8)$ \\
Employed, n (\%) & $400(20.7)$ & $297(18.9)$ \\
Living with someone, n (\%) & $1,397(72.2)$ & $1,027(65.3)$ \\
Smoking, n (\%) & & \\
$\quad$ Never & $626(32.4)$ & $553(35.2)$ \\
Former & $1,137(58.8)$ & $806(51.2)$ \\
Current & $170(8.8)$ & $204(13.0)$ \\
Alcohol, grams/day & $12.8(15.2)$ & $10.1(13.1)$ \\
BMI & $25.1(4.6)$ & $25.5(4.9)$ \\
Diet quality score (0-14) & $6.9(1.8)$ & $6.9(2.0)$ \\
Comorbidities, n (\%) & & \\
0 & $1,138(58.8)$ & $926(58.9)$ \\
1 & $623(32.2)$ & $521(33.1)$ \\
2 & $153(7.9)$ & $116(7.4)$ \\
\hline 3 & $20(1.0)$ & $9(0.6)$ \\
\hline Use of sleep medication, n (\%) & $282(14.6)$ & $123(7.8)^{\mathrm{a}}$ \\
\hline
\end{tabular}

Note: Numbers are mean (SD), unless otherwise stated.

a $55 \%$ missing.

LIPA, light intensity physical activity; MVPA, moderate-to-vigorous physical activity; VAS, visual analogue scale. 
Appendix Table 2. Sex-Age Adjusted Model for Estimated Differences in EQ-5D-3L Index Score and VAS Score Associated With 30-minute Reallocations Between Activity Behaviors ( $\mathrm{n}=1,934)$

\begin{tabular}{|c|c|c|c|c|}
\hline Variable & $\begin{array}{c}\text { LIPA } \\
\text { Delta }(95 \% \mathrm{CI}) \\
\end{array}$ & $\begin{array}{c}\text { MVPA } \\
\text { Delta }(95 \% \mathrm{CI}) \\
\end{array}$ & $\begin{array}{c}\text { SB } \\
\text { Delta }(95 \% \mathrm{CI}) \\
\end{array}$ & $\begin{array}{c}\text { Sleep } \\
\text { Delta }(95 \% \text { CI }) \\
\end{array}$ \\
\hline \multicolumn{5}{|c|}{ EQ-5D-3L index score } \\
\hline LIPA & & $-0.06(-0.09,-0.03)$ & $-0.01(-0.02,0.002)$ & $-0.01(-0.02,0.002)$ \\
\hline MVPA & $0.05(0.02,0.07)$ & & $0.04(0.02,0.05)$ & $0.04(0.02,0.05)$ \\
\hline SB & $0.01(-0.002,0.02)$ & $-0.05(-0.07,-0.03)$ & & $0.0001(-0.003,0.003)$ \\
\hline Sleep & $0.01(-0.002,0.02)$ & $-0.05(-0.07,-0.03)$ & $0.0001(-0.003,0.003)$ & \\
\hline \multicolumn{5}{|l|}{ VAS score } \\
\hline LIPA & & $-4.38(-6.88,-1.88)$ & $-0.78(-1.76,0.19)$ & $-0.69(-1.67,0.28)$ \\
\hline MVPA & $3.41(1.24,5.59)$ & & $2.44(1.35,3.53)$ & $2.53(1.39,3.67)$ \\
\hline SB & $0.98(-0.20,2.16)$ & $-3.59(-5.23,-1.96)$ & & $0.09(-0.20,0.39)$ \\
\hline Sleep & $0.89(-0.28,2.06)$ & $-3.68(-5.35,-2.01)$ & $-0.08(-0.36,0.20)$ & \\
\hline
\end{tabular}

Note: Boldface indicates statistical significance $(p<0.05)$. Estimates are obtained from compositional data analysis and reflect difference in EQ-5D-3L index score and VAS score for the reallocation of time from the behavior in the column to the behavior in the row, while keeping the time spent in other activities constant. Reallocations of behaviors are relative to the mean activity composition of the study population. Higher EQ-5D-3L index score and VAS score indicate higher quality of life.

LIPA, light intensity physical activity; MVPA, moderate-to-vigorous physical activity; SB, sedentary behavior; VAS, visual analogue scale. 
Appendix Table 3. Associations of Reallocations Between Activity Behaviors and the EQ-5D-3L Index Score, Stratified by Sex $(\mathrm{n}=1,934)$

\begin{tabular}{|c|c|c|c|c|}
\hline Variable & $\begin{array}{c}\text { LIPA } \\
\text { Delta }(95 \% \text { CI })\end{array}$ & $\begin{array}{c}\text { MVPA } \\
\text { Delta }(95 \% \text { CI })\end{array}$ & $\begin{array}{c}\text { SB } \\
\text { Delta }(95 \% \text { CI })\end{array}$ & $\begin{array}{c}\text { Sleep } \\
\text { Delta }(95 \% \text { CI) }\end{array}$ \\
\hline \multicolumn{5}{|c|}{ Men $(n=938)$} \\
\hline LIPA & & $-0.06(-0.09,-0.03)$ & $-0.01(-0.03,0.001)$ & $-0.01(-0.03,-0.001)$ \\
\hline MVPA & $0.05(0.02,0.08)$ & & $0.03(0.02,0.05)$ & $0.03(0.02,0.05)$ \\
\hline SB & $0.02(0.001,0.03)$ & $-0.05(-0.07,-0.02)$ & & $-0.001(-0.004,0.001)$ \\
\hline Sleep & $0.02(0.001,0.03)$ & $-0.05(-0.07,-0.02)$ & $0.001(-0.004,0.001)$ & \\
\hline \multicolumn{5}{|c|}{ Women $(n=996)$} \\
\hline LIPA & & $-0.04(-0.09,0.001)$ & $-0.007(-0.02,0.009)$ & $-0.004(-0.02 .0 .01)$ \\
\hline MVPA & $0.03(-0.005,0.07)$ & & $0.02(0.005,0.05)$ & $0.03(0.01,0.05)$ \\
\hline SB & $0.01(-0.01,0.03)$ & $-0.04(-0.06,-0.01)$ & & $0.003(-0.002,0.01)$ \\
\hline Sleep & $0.006(-0.02,0.03)$ & $-0.04(-0.07,-0.01)$ & $-0.003(-0.01,0.002)$ & \\
\hline
\end{tabular}

Note: Boldface indicates statistical significance $(p<0.05)$. Estimates are obtained from compositional data analysis and reflect difference in EQ-5D-3L index score and VAS score for the reallocation of time from the behavior in the column to the behavior in the row, while keeping the time spent in other activities constant. Reallocations of behaviors are relative to the mean activity composition of the study population. Higher EQ-5D-3L index score and VAS score indicate higher quality of life. Models are adjusted for age, educational status, marital status, living situation, job status, smoking, alcohol intake, dietary guidelines score, BMI, comorbidity score and the use of sleep medication.

LIPA, light intensity physical activity; MVPA, moderate-to-vigorous physical activity; SB, sedentary behavior; VAS, visual analogue scale. 
Appendix Table 4. Associations of Reallocations Between Activity Behaviors and the EQ-5D-3L Index Score, Stratified by Median Age $(n=1,934)$

\begin{tabular}{|c|c|c|c|c|}
\hline Variable & $\begin{array}{c}\text { LIPA } \\
\text { Delta }(95 \% \text { CI) }\end{array}$ & $\begin{array}{c}\text { MVPA } \\
\text { Delta }(95 \% \text { CI) }\end{array}$ & $\begin{array}{c}\text { SB } \\
\text { Delta }(95 \% \text { CI })\end{array}$ & $\begin{array}{c}\text { Sleep } \\
\text { Delta }(95 \% \text { CI })\end{array}$ \\
\hline \multicolumn{5}{|c|}{$<72$ years $(n=921)$} \\
\hline LIPA & & $-0.04(-0.07,-0.01)$ & $-0.02(-0.03,-0.002)$ & $-0.01(-0.03,0.002)$ \\
\hline MVPA & $0.04(0.01,0.07)$ & & $0.02(0.004,0.03)$ & $0.02(0.01,0.04)$ \\
\hline SB & $0.02(0.003,0.04)$ & $-0.03(-0.05,-0.01)$ & & $0.004(-0.001,0.01)$ \\
\hline Sleep & $0.02(-0.001,0.03)$ & $-0.03(-0.05,-0.01)$ & $-0.004(-0.01,0.001)$ & \\
\hline \multicolumn{5}{|c|}{$\geq 72$ years $(n=1,013)$} \\
\hline LIPA & & $-0.07(-0.11,-0.02)$ & $-0.004(-0.02,0.01)$ & $-0.004(-0.02,0.01)$ \\
\hline MVPA & $0.05(0.01,0.08)$ & & $0.04(0.02,0.06)$ & $0.04(0.02,0.06)$ \\
\hline SB & $0.01(-0.01,0.02)$ & $-0.06(-0.09,-0.03)$ & & $-0.001(-0.005,0.005)$ \\
\hline Sleep & $0.001(-0.01,0.02)$ & $-0.06(-0.09,-0.03)$ & $0.001(-0.004,0.005)$ & \\
\hline
\end{tabular}

Note: Boldface indicates statistical significance $(p<0.05)$. Estimates are obtained from compositional data analysis and reflect difference in EQ-5D-3L index score and VAS score for the reallocation of time from the behavior in the column to the behavior in the row, while keeping the time spent in other activities constant. Reallocations of behaviors are relative to the mean activity composition of the study population. Higher EQ-5D-3L index score and VAS score indicate higher quality of life. Models are adjusted for sex, educational status, marital status, living situation, job status, smoking, alcohol intake, dietary guidelines score, BMI, comorbidity score and the use of sleep medication.

LIPA, light intensity physical activity; MVPA, moderate-to-vigorous physical activity; SB, sedentary behavior; VAS, visual analogue scale. 
Appendix Table 5. Associations of Reallocations Between Activity Behaviors and the EQ-5D-3L Index Score, Stratified by Median Moderate-to-Vigorous Activity Level $(\mathrm{n}=1,934)$

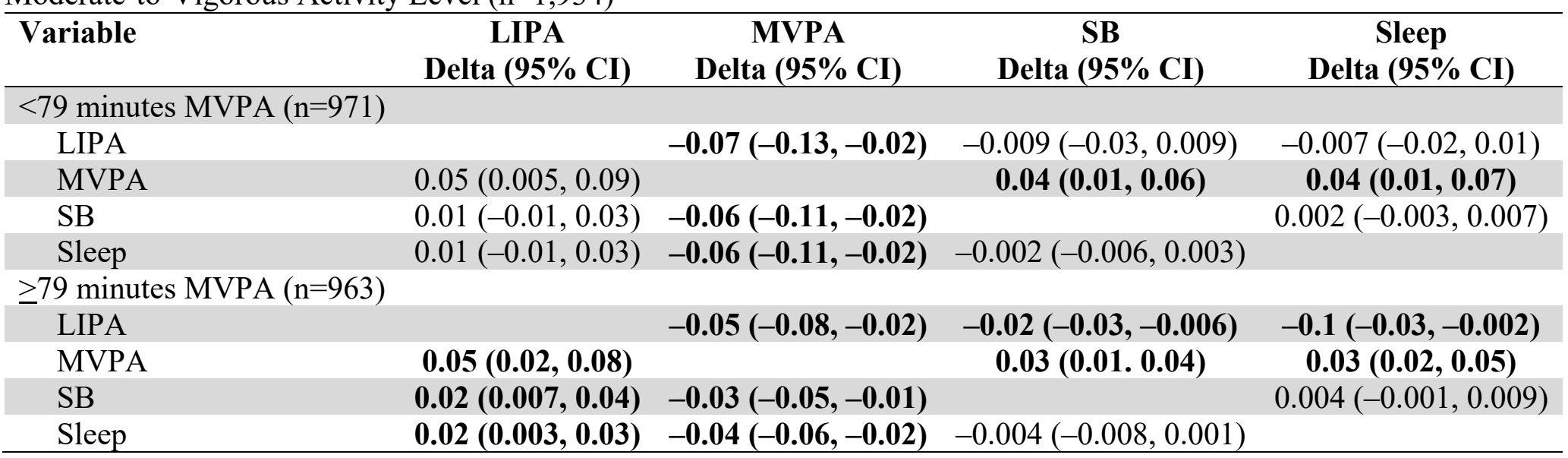

Note: Boldface indicates statistical significance $(p<0.05)$. Estimates are obtained from compositional data analysis and reflect difference in EQ-5D-3L index score and VAS score for the reallocation of time from the behavior in the column to the behavior in the row, while keeping the time spent in other activities constant. Reallocations of behaviors are relative to the mean activity composition of the study population. Higher EQ-5D-3L index score and VAS score indicate higher quality of life. Models are adjusted for sex, educational status, marital status, living situation, job status, smoking, alcohol intake, dietary guidelines score, BMI, comorbidity score and the use of sleep medication.

LIPA, light intensity physical activity; MVPA, moderate-to-vigorous physical activity; SB, sedentary behavior; VAS, visual analogue scale. 
Appendix Table 6. Associations of Reallocations Between Activity Behaviors and the VAS Score, Stratified by Sex $(\mathrm{n}=1,934)$

\begin{tabular}{|c|c|c|c|c|}
\hline Variable & $\begin{array}{c}\text { LIPA } \\
\text { Delta }(95 \% \text { CI })\end{array}$ & $\begin{array}{c}\text { MVPA } \\
\text { Delta }(95 \% \text { CI })\end{array}$ & $\begin{array}{c}\text { SB } \\
\text { Delta }(95 \% \text { CI })\end{array}$ & $\begin{array}{c}\text { Sleep } \\
\text { Delta }(95 \% \text { CI })\end{array}$ \\
\hline \multicolumn{5}{|c|}{$\operatorname{Men}(n=938)$} \\
\hline LIPA & & $-5.15(-8.80,-1.50)$ & $-1.24(-2.65,0.16)$ & $-0.92(-2.31,0.47)$ \\
\hline MVPA & $4.10(0.96,7.25)$ & & $2.58(0.99,4.18)$ & $2.90(1.23,4.58)$ \\
\hline SB & $1.52(-0.19,3.23)$ & $-3.90(-6.32,-1.49)$ & & $0.32(-0.09,0.74)$ \\
\hline Sleep & $1.22(-0.47,2.90)$ & $-4.21(-6.68,-1.73)$ & $-0.30(-0.70,0.10)$ & \\
\hline \multicolumn{5}{|c|}{ Women $(n=996)$} \\
\hline LIPA & & $-2.42(-5.96,1.11)$ & $-0.48(-1.85,0.90)$ & $-0.35(-1.74,1.03)$ \\
\hline MVPA & $1.91(-1.17,4.99)$ & & $1.33(-0.25,2.90)$ & $1.45(-0.16,3.06)$ \\
\hline SB & $0.58(-1.08,2.24)$ & $-1.94(-4.27,0.38)$ & & $0.12(-0.31,0.55)$ \\
\hline Sleep & $0.47(-1.19,2.13)$ & $-2.06(-4.40,0.29)$ & $-0.11(-0.52,0.30)$ & \\
\hline
\end{tabular}

Note: Boldface indicates statistical significance $(p<0.05)$. Estimates are obtained from compositional data analysis and reflect difference in EQ-5D-3L index score and VAS score for the reallocation of time from the behavior in the column to the behavior in the row, while keeping the time spent in other activities constant. Reallocations of behaviors are relative to the mean activity composition of the study population. Higher EQ-5D-3L index score and VAS score indicate higher quality of life. Models are adjusted for age, educational status, marital status, living situation, job status, smoking, alcohol intake, dietary guidelines score, BMI, comorbidity score and the use of sleep medication.

LIPA, light intensity physical activity; MVPA, moderate-to-vigorous physical activity; SB, sedentary behavior; VAS, visual analogue scale. 
Appendix Table 7. Associations of Reallocations Between Activity Behaviors and the VAS Score, Stratified by Median Age $(\mathrm{n}=1,934)$

\begin{tabular}{|c|c|c|c|c|}
\hline Variable & $\begin{array}{c}\text { LIPA } \\
\text { Delta }(95 \% \text { CI) }\end{array}$ & $\begin{array}{c}\text { MVPA } \\
\text { Delta }(95 \% \text { CI })\end{array}$ & $\begin{array}{c}\text { SB } \\
\text { Delta }(95 \% \text { CI })\end{array}$ & $\begin{array}{c}\text { Sleep } \\
\text { Delta }(95 \% \mathrm{CI})\end{array}$ \\
\hline \multicolumn{5}{|c|}{$<72$ years $(n=921)$} \\
\hline LIPA & & $-2.87(-6.57,0.83)$ & $-1.07(-2.66,0.52)$ & $-0.73(-2.32,0.87)$ \\
\hline MVPA & $2.52(-0.84,5.89)$ & & $1.25(-0.39,2.88)$ & $1.59(-0.11,3.28)$ \\
\hline SB & $1.27(-0.64,3.19)$ & $-1.81(-4.11,0.50)$ & & $0.34(-0.17,0.84)$ \\
\hline Sleep & $0.95(-0.96,2.86)$ & $-2.13(-4.48,0.23)$ & $-0.32(-0.81,0.16)$ & \\
\hline \multicolumn{5}{|c|}{$\geq 72$ years $(\mathrm{n}=1,013)$} \\
\hline LIPA & & $-2.94(-6.09,0.21)$ & $-0.21(-1.37,0.95)$ & $-0.08(-1.22,1.05)$ \\
\hline MVPA & $2.02(-0.60,4.64)$ & & $1.75(0.40,3.10)$ & $1.88(0.47,3.29)$ \\
\hline SB & $0.27(-1.14,1.68)$ & $-2.73(-4.86,-0.59)$ & & $0.13(-0.22,0.47)$ \\
\hline Sleep & $0.16(-1.22,1.54)$ & $-2.84(-5.03,-0.65)$ & $-0.11(-0.45,0.22)$ & \\
\hline
\end{tabular}

Note: Boldface indicates statistical significance $(p<0.05)$. Estimates are obtained from compositional data analysis and reflect difference in EQ-5D-3L index score and VAS score for the reallocation of time from the behavior in the column to the behavior in the row, while keeping the time spent in other activities constant. Reallocations of behaviors are relative to the mean activity composition of the study population. Higher EQ-5D-3L index score and VAS score indicate higher quality of life. Models are adjusted for sex, educational status, marital status, living situation, job status, smoking, alcohol intake, dietary guidelines score, BMI, comorbidity score and the use of sleep medication.

LIPA, light intensity physical activity; MVPA, moderate-to-vigorous physical activity; SB, sedentary behavior; VAS, visual analogue scale. 
Appendix Table 8. Associations of Reallocations Between Activity Behaviors and the VAS Score, Stratified by Median Moderate-toVigorous Activity Level $(\mathrm{n}=1,934)$

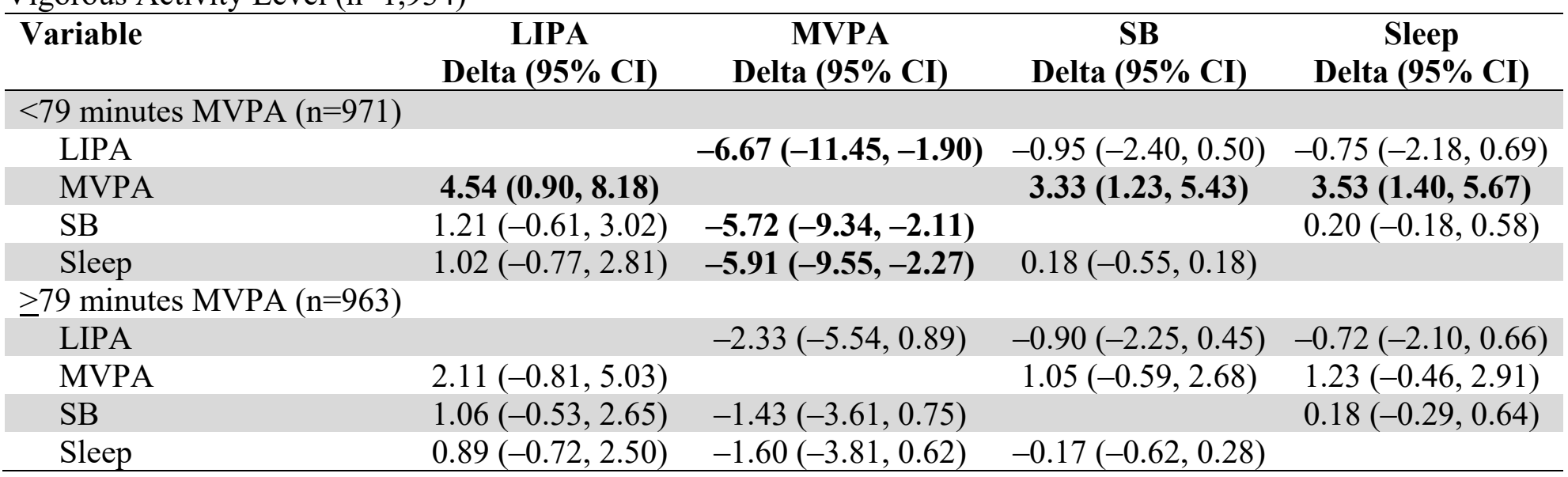

Note: Boldface indicates statistical significance $(p<0.05)$. Estimates are obtained from compositional data analysis and reflect difference in EQ-5D-3L index score and VAS score for the reallocation of time from the behavior in the column to the behavior in the row, while keeping the time spent in other activities constant. Reallocations of behaviors are relative to the mean activity composition of the study population. Higher EQ-5D-3L index score and VAS score indicate higher quality of life. Models are adjusted for sex, educational status, marital status, living situation, job status, smoking, alcohol intake, dietary guidelines score, BMI, comorbidity score and the use of sleep medication.

LIPA, light intensity physical activity; MVPA, moderate-to-vigorous physical activity; SB, sedentary behavior; VAS, visual analogue scale. 